\title{
Intra-, Inter-, and Extra-container Path Loss for Shipping Container Monitoring Systems
}

\author{
Emmeric Tanghe, Wout Joseph, Member, IEEE, Peter Ruckebusch, Luc Martens, Member, IEEE, and \\ Ingrid Moerman, Member, IEEE
}

\begin{abstract}
This paper presents empirical path loss models for an environment of stacked shipping containers. Specifically, a system for wireless monitoring of containers is considered for which three different types of wireless links are identified, namely intra-, inter-, and extra-container links. Furthermore, the intercontainer link is investigated for the two most common types of container stacking: row and block stacking. Intra- and intercontainer path loss is investigated at IEEE 802.15.4 frequencies of 433, 868, and $2400 \mathrm{MHz}$. Extra-container path loss is examined at GSM/UMTS frequencies of 900, 1850, and 2100 MHz. Distancedependent path loss models are proposed for the inter- and extracontainer links (high correlation coefficients between 0.76 and 0.86). The resulting path loss models can be used in link budget calculations for container monitoring systems.
\end{abstract}

Index Terms-Container, path loss, MoCo

\section{INTRODUCTION}

One of the major tasks of supply chain management is to follow goods, stored in shipping containers, from origin to final destination. This paper presents path loss modeling for a general wireless architecture for container monitoring and tracking, developed within the IBBT MoCo project. The architecture is based around so-called MoCo (for Monitoring of Containers) wireless devices. A MoCo device is mounted inside a container and is connected to an antenna outside through the container's ventilation holes (which are near the container's ceiling, at a height of about $2.5 \mathrm{~m}$ ). These ventilation holes are almost always covered by a plastic cap, which is helpful to cover and protect the antenna. A MoCo device has three main functionalities: (i) it can wirelessly gather data from sensors inside the container that measure transport parameters such as temperature, humidity, shock, and door status, (ii) it can relay that data to other MoCo devices attached to other containers, and (iii) it can send data to a cloud for storage and retrieval. Obtaining enough wireless link budget for these functionalities is challenging because of the highly metallic (shielding) nature of the container environment. Because of the particularity of the container environment, well-known path loss models for outdoor environments (e.g. COST 231

The IBBT MoCo is a project co-funded by IBBT (Interdisciplinary institute for Technology), a research institute founded by the Flemish Government. Companies and organizations involved in the project are Multicap and Rmoni Wireless, with project support of IWT. This research is also partly funded by the Fund for Scientific Research - Flanders (FWO-V, Belgium) project G.0325.11N. W. Joseph is a Post-Doctoral Fellow of the FWO-V (Research Foundation - Flanders).

E. Tanghe, W. Joseph, P. Ruckebusch, L. Martens, and I. Moerman are with IBBT-INTEC-Ugent (Department of Information Technology - Ghent University), Gaston Crommenlaan 8 box 201, B-9050 Ghent, Belgium, Email: emmeric.tanghe@intec.UGent.be.
Walfisch-Ikegami [1]) are an unsatisfactory fit for empirical path loss around containers [2].

Path loss models for container environments have only very recently become available in literature. In [3], path models models based on measurements in a container terminal are proposed for frequencies between 1 and $4 \mathrm{GHz}$ and for different antenna heights. In [4] and [5], path loss is investigated for Radio Frequency Identification (RFID) tags attached to containers. Finally, the work in [6] takes into account the shielding and waveguiding effects of metallic objects to improve location estimation accuracy in container environments. This paper presents path loss modelling in a container environments for different types of wireless links, frequencies, and container stacking configurations. In contrast to [3], path loss is investigated for containers in the near field of the transmitting and/or the receiving antenna. This is to reflect the actual operating scenario wherein the MoCo device's antenna is attached directly to the container surface. While [4] and [5] focus on path loss in and around a single container, this work is intended for larger stacks of containers.

\section{PROpagation SCEnARIOS}

We distinguish between three types of wireless links between sensors, MoCo devices, and the cloud. Each link type handles one of the MoCo device's main functionalities:

- Intra-container link: a sensor inside the container transmits sensory data to the MoCo device's antenna which is attached outside to that container (IEEE 802.15.4).

- Inter-container link: relaying of data between MoCo device antennas attached to different containers (IEEE 802.15.4).

- Extra-container link: A MoCo device's antenna sends data to the cloud (GSM, UMTS).

In the above list, it is also mentioned which communication standard(s) is (are) suitable for implementing each link.

\section{MEASUREMENT SETUP AND DATA PROCESSING}

The measurement setup consists of a transmitting and a receiving part. At the transmitter, a signal generator (Rohde \& Schwarz SMP22) creates a continuous wave which is fed to the transmitting antenna Tx. At the receiving end, a spectrum analyzer (Hewlett-Packard 8561B) samples the power at the receiving antenna $\mathrm{Rx}$. As Tx and Rx, vertically polarized halfwave dipole antennas are used. In case the measured link is for one or both of the antennas attached to a container surface, a styrofoam spacer of thickness $1 \mathrm{~cm}$ is placed between the 
antenna and the container. Path loss $P L$ in $\mathrm{dB}$ is calculated as:

$$
P L=P_{T x}-L_{T x}-L_{R x}-P_{R x}
$$

In (1), $P_{T x}$ is the transmit power $(20 \mathrm{dBm}), L_{T x}$ and $L_{R x}$ are the $\mathrm{Tx}$ and $\mathrm{Rx}$ antenna feeder losses in $\mathrm{dB}$, and $P_{R x}$ is the received power in $\mathrm{dBm}$. It is important to note that the usual calculation of path loss also includes terms in the right-hand side of (1) which exclude the gains of the measurement antennas. This was however not done here: due to the proximity of the metallic container surface, antenna effects cannot be separated from the wireless propagation loss. This is highly similar to the definition of path loss for on-body propagation channels, where the proximity of the human body also prevents exclusion of antenna radiation patterns from the wireless propagation loss [7].

\section{REsults}

\section{A. Intra-container link}

In the MoCo architecture, the intra-container link adopts the IEEE 802.15.4 standard. Penetration loss from outside to inside a container is investigated for three frequencies targeted by IEEE 802.15.4: 433, 868, and $2400 \mathrm{MHz}$. The antenna placement for the intra-container measurements is shown in Fig. 1. The Tx is mounted outside the container on the ventilation holes nearest to the container door. The $\mathrm{Rx}$ is installed alternatingly at two different locations inside the container. The first $\mathrm{Rx}$ location $\left(\mathrm{Rx}_{1}\right)$ is inside on the ventilation holes, directly facing the $\mathrm{Tx}$ on the outside. The second $\mathrm{Rx}$ location $\left(\mathrm{Rx}_{2}\right)$ is inside at the container's center.

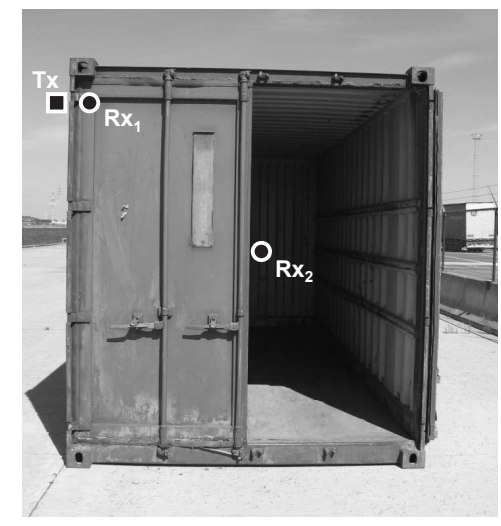

Figure 1. Intra-container scenario

Intra-container measurements were carried out for two containers, one 20' (20 feet) container and one 40' container. The designations $20^{\prime}$ and $40^{\prime}$ refer to the (standardized) length of the container. Unlike the 40' container, the 20' container is an older type that was not fitted out with ventilation holes, so $\mathrm{Tx}$ and $\mathrm{Rx}_{1}$ were mounted where the ventilation holes are expected to be on this type of container. Both containers were empty during measurements and their doors were closed. For each of the two Tx-Rx links, 200 samples of the received power $P_{R x}$ are recorded. These samples are used to calculate 200 path loss (penetration loss) values using (1). Fig. 2 shows bar plots of the maximum (worst-case) penetration loss over the 200 samples for each frequency, container type, and Tx-Rx link. A transparent bar means that the maximum penetration loss exceeds the spectrum analyzer's sensitivity, and the value mentioned on top of the bar is the maximum measurable penetration loss. In Fig. 2, no clear relation between penetration loss and frequency is observed. This relation can be complicated and non-monotonic due to variation of the constitutive parameters of container steel with frequency, container geometry, and the presence of ventilation holes [8].

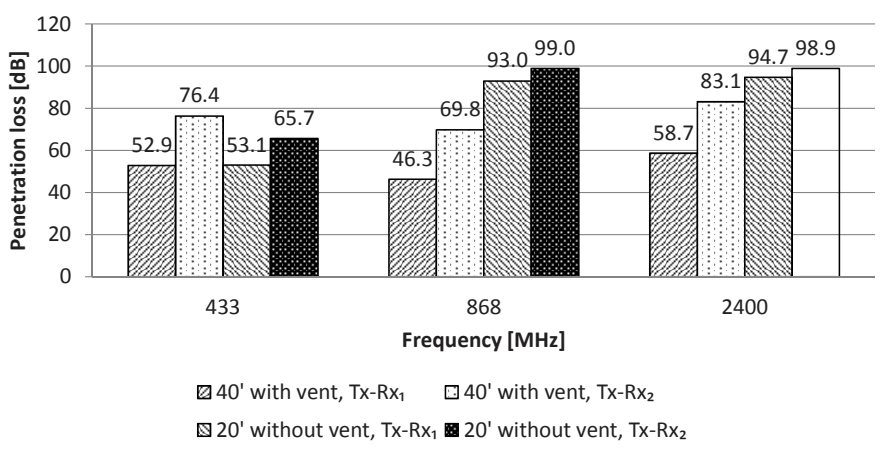

Figure 2. Maximum penetration loss for the intra-container link

The penetration losses in Fig. 2 are compared to typical values of the maximum path loss (corresponding to $10 \%$ packet error rate) of wireless sensor modules. A commercial sensor module operating at 433 and $868 \mathrm{MHz}$ is the Amber Wireless AMB8423, which has a nominal maximum path loss of $105 \mathrm{~dB}$. The Tmote Sky is a sensor module operating at $2400 \mathrm{MHz}$ with a nominal maximum path loss of $94 \mathrm{~dB}$. For the 40 ' container, the measured maximum penetration loss in Fig. 2 is sufficiently limited to allow for a reliable intracontainer link. This is the case for all three frequencies, and for both $\mathrm{Tx}-\mathrm{Rx}$ links. The penetration loss for $\mathrm{Tx}-\mathrm{Rx}_{2}$ is about $27 \mathrm{~dB}$ larger than for $\mathrm{Tx}-\mathrm{Rx}_{1}$. For the 20' container, it can be concluded that a reliable intra-container link is not possible for 868 and $2400 \mathrm{MHz}$. A reliable link is however possible for $433 \mathrm{MHz}$.

\section{B. Inter-container link}

Path loss for the inter-container link is investigated for the IEEE 802.15.4 frequencies of 433, 868, and 2400 MHz. The measurements are carried out for two common types of container stacking: row and block stacking.

1) Container row stacking: The measurements are carried out along the row of 4 containers (total length of $42.7 \mathrm{~m}$ ) shown in Fig. 3. The Tx is mounted on the ventilation holes near the top of the first container. The Rx is then moved in steps of $0.5 \mathrm{~m}$ along the top of the container row at the same height above ground level as the Tx. At each Rx location, the median of 200 samples of received power is recorded. In total, $83 \mathrm{Rx}$ locations are measured per frequency. Measured path loss in $\mathrm{dB}$ is found to correlate well with logarithmic distance (average correlation of 0.78 over the three frequencies). Path loss $P L$ (in $\mathrm{dB}$ ) is fitted to the following model as function of distance $d$ (in $\mathrm{m}$ ) between Tx and Rx:

$$
P L(d)=b_{0}+b_{1} \cdot 10 \log _{10}(d)+\chi_{s}
$$




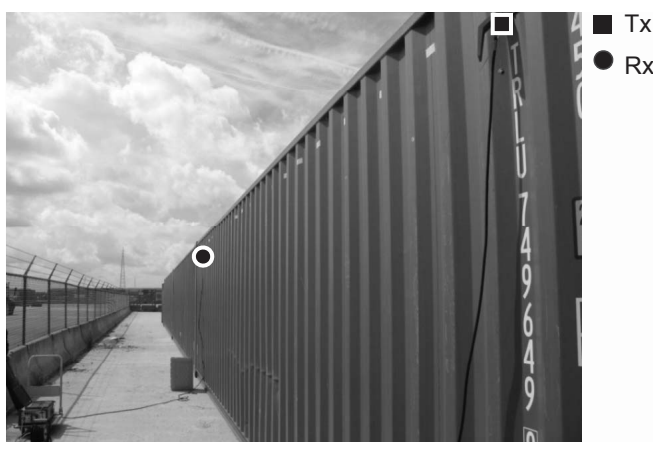

Figure 3. Inter-container scenario for row stacking

In (2), $b_{0}$ and $b_{1}$ are regression parameters $\left(b_{1}\right.$ is the path loss exponent) and $\chi_{s}$ is a zero-mean random variable that accounts for shadow fading. $\chi_{s}$ assumes a normal distribution with standard deviation $\sigma_{s}$. Fig. 4 shows measured path loss versus distance for the three frequencies. Also shown is the fitted regression line $b_{0}+b_{1} \cdot 10 \log _{10}(d)$ for each frequency. Table I lists estimated values for the parameters of (2), obtained by least-squares fitting.

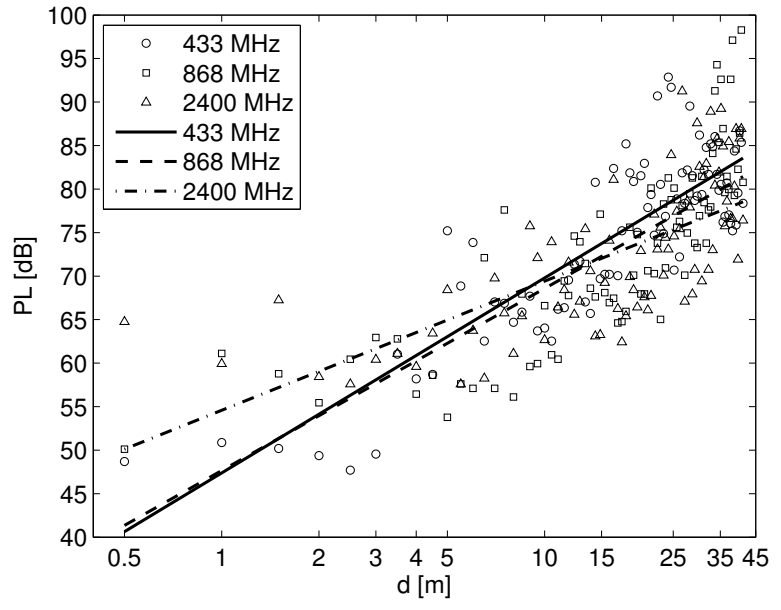

Figure 4. Path loss versus distance for the inter-container link (row stacking)

\begin{tabular}{|c|ccc|}
\hline frequency $[\mathrm{MHz}]$ & $b_{0}[\mathrm{~dB}]$ & $b_{1}[-]$ & $\sigma_{s}[\mathrm{~dB}]$ \\
\hline 433 & 47.38 & 2.24 & 5.49 \\
868 & 47.64 & 2.09 & 6.56 \\
2400 & 54.58 & 1.48 & 6.19 \\
\hline
\end{tabular}

Table I

INTER-CONTAINER PATH LOSS MODEL PARAMETERS (ROW STACKING)

The $95 \%$ confidence bounds on the vertical location of the regression lines are at most $\pm 2.80 \mathrm{~dB}$ over all frequencies and distances. Taking this uncertainty into account, it can be concluded from Fig. 4 that path loss is nearly identical for all frequencies at large distances. At small distances, path loss is similar for 433 and $868 \mathrm{MHz}$, but is higher for $2400 \mathrm{MHz}$. This can attributed to the periodically ribbed structure of container surfaces (period $=27 \mathrm{~cm}$, Fig. 3). At smaller distances, surface waves along the container dominate propagation. In contrast to 433 and $868 \mathrm{MHz}$, the wavelength at $2400 \mathrm{MHz}$ is smaller than the ribbed structure's period, which means this frequency is more prone to diffraction losses at the rib edges. This results in a comparatively gentler slope for the regression line at $2400 \mathrm{MHz}$ and a path loss exponent $b_{1}$ smaller than 2 .

2) Container block stacking: The measurements were carried out on a three-dimensional stack of sixteen 20' containers (4 long, 2 wide, and 2 high), shown schematically Fig. 5. Measurements are done for two scenarios. In the first and second scenario, the antennas of the MoCo devices are mounted outside on the containers' ventilation holes (black symbols) and doors (white symbols), respectively. For both scenarios, path loss is measured between the Tx on container 1 (squares) and the Rx's on the other containers (circles). For each Tx-Rx link, the median of 300 samples of received power is recorded.

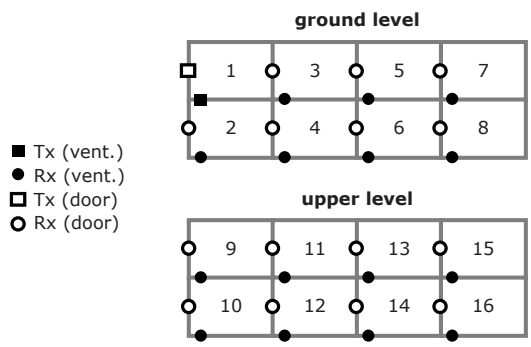

Figure 5. Inter-container scenario for block stacking

Fig. 6 shows measured path loss versus distance for the three frequencies and both scenarios. The path loss samples are fitted to the regression model in (2): Fig. 6 shows the fitted regression lines and Table II lists the estimated regression parameters. The average correlation between path loss and logarithmic distance for the six fits in Fig. 6 is 0.76. The $95 \%$ confidence bounds on the vertical location of the six regression lines are $\pm 4.94 \mathrm{~dB}$ on average over all frequencies and distances.

For the vent-mounted scenario, path loss is nearly the same at 433 and $868 \mathrm{MHz}$ and comparatively smaller at $2400 \mathrm{MHz}$. Propagation between $\mathrm{Tx}$ and $\mathrm{Rx}$ for this scenario occurs mainly along the small gaps between two containers (with widths of around $10 \mathrm{~cm}$ ), due to the fact that both Tx and $\mathrm{Rx}$ are closed in between containers. Propagation in small gaps is less lossy at $2400 \mathrm{MHz}$ due to the smaller wavelength.

For the door-mounted scenario, path loss is similar at all frequencies for smaller distances and relatively smaller at $433 \mathrm{MHz}$ for larger distances. Since the Tx for the doormounted scenario faces free space on one side (Fig. 5), part of the propagation path runs in the free space around the container stack, and part of the path runs in the gaps between the containers in order to reach the Rx. For smaller distances, a larger part of the path runs in the gaps where $433 \mathrm{MHz}$ experiences high loss (large wavelength in small gaps). For larger distances, a larger part of the path runs in free space where $433 \mathrm{MHz}$ experiences low loss (low frequency in free space). This difference in relative path loss between smaller and larger distances causes the regression line at $433 \mathrm{MHz}$ to tilt and results in a path loss exponent $b_{1}$ smaller than 2 .

\section{Extra-container link}

In the MoCo architecture, the extra-container link is implemented with GSM and/or UMTS. Path loss is investigated for 

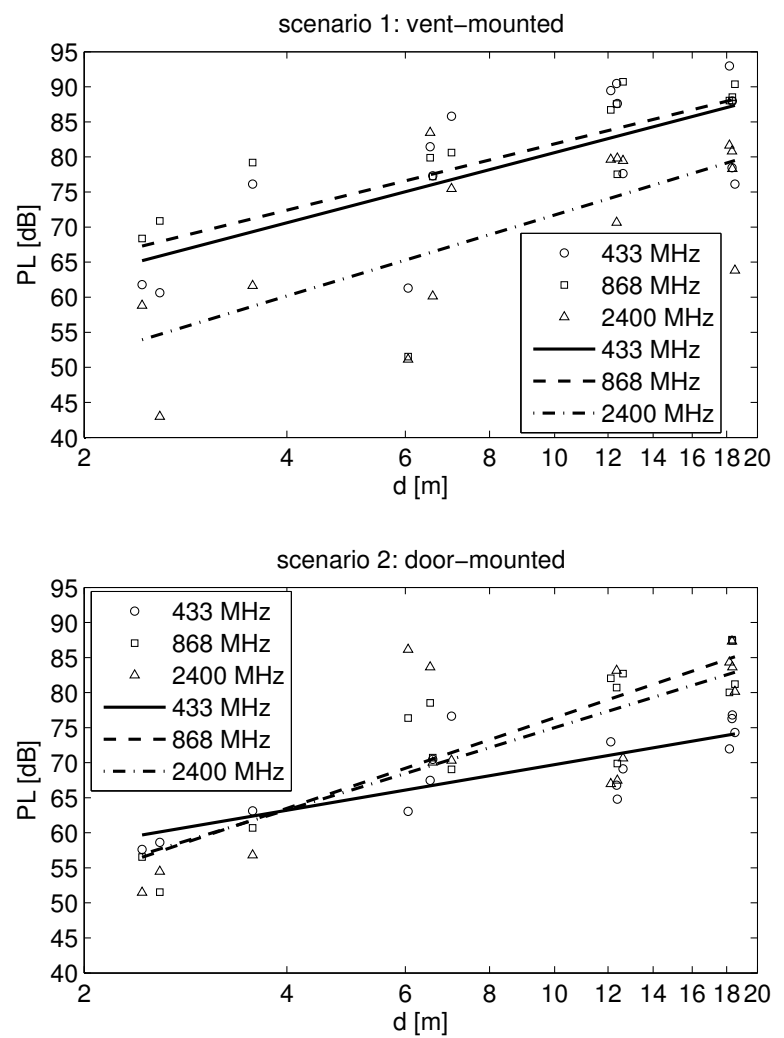

Figure 6. Path loss versus distance for the inter-container link (block stacking): vent-mounted and door-mounted scenario

\begin{tabular}{|c|ccc|ccc|}
\hline & \multicolumn{3}{|c|}{ vent-mounted } & \multicolumn{3}{c|}{ door-mounted } \\
frequency & $b_{0}$ & $b_{1}$ & $\sigma_{s}$ & $b_{0}$ & $b_{1}$ & $\sigma_{s}$ \\
{$[\mathrm{MHz}]$} & {$[\mathrm{dB}]$} & {$[-]$} & {$[\mathrm{dB}]$} & {$[\mathrm{dB}]$} & {$[-]$} & {$[\mathrm{dB}]$} \\
\hline 433 & 55.46 & 2.52 & 7.76 & 53.36 & 1.64 & 3.98 \\
868 & 58.10 & 2.38 & 7.98 & 43.93 & 3.25 & 4.94 \\
2400 & 42.69 & 2.90 & 9.30 & 45.50 & 2.95 & 8.33 \\
\hline
\end{tabular}

Table II

INTER-CONTAINER PATH LOSS MODEL PARAMETERS (BLOCK STACKING)

the GSM/UMTS frequencies of 900, 1850, and $2100 \mathrm{MHz}$. Path loss measurements for the extra-container link were carried out outside a large stack of 40' containers. Fig. 7 presents a top-down view of the container configuration. The stack varies in height from 4 to 7 containers. The pathways between the container rows are about $1.5 \mathrm{~m}$ wide. The $\mathrm{Tx}$ is mounted near the ventilation holes at the top of one of the containers on the ground level. The Rx is attached to a mast on a cart and is at the same height as the Tx. The cart is pushed along several tracks outside the stack (the striped area in Fig. 7) while the spectrum analyzer continuously samples the received power.

Extra-container path loss is smoothed out using a sliding window of length $3 \mathrm{~m}$ to remove small-scale fading. Per frequency, on average 973 smoothed path loss samples are obtained. These path loss samples cover distances between the Tx and Rx from 10 up to $63 \mathrm{~m}$. Path loss is found to correlate well with logarithmic distance (frequency-average correlation coefficient of 0.86) and is therefore fitted to the model in (2). Table III lists the estimated parameters of (2). It is observed that the path loss exponent $b_{1}$ is smaller than 2 for all three frequencies. This points to a waveguiding effect. In Fig. 7,

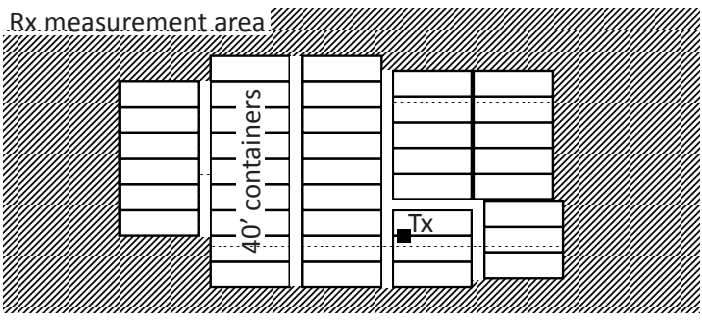

Figure 7. Extra-container scenario

\begin{tabular}{|c|ccc|}
\hline frequency $[\mathrm{MHz}]$ & $b_{0}[\mathrm{~dB}]$ & $b_{1}[-]$ & $\sigma_{s}[\mathrm{~dB}]$ \\
\hline 900 & 70.19 & 1.82 & 3.22 \\
1850 & 85.34 & 1.27 & 3.17 \\
2100 & 85.88 & 1.22 & 3.30 \\
\hline
\end{tabular}

Table III

EXTRA-CONTAINER PATH LOSS MODEL PARAMETERS

part of the propagation path between Tx and Rx runs along the narrow pathways flanked by the highly-reflective container stacks (i.e. the white space between the 40' containers). These pathways function as a low-loss waveguide.

\section{Conclusions}

In this work, path loss has been investigated for three types of wireless links in a shipping container environment: intra-, inter-, and extra-container links. The intra- and inter-container links are investigated for the IEEE 802.15.4 frequencies of 433, 868, and $2400 \mathrm{MHz}$. Intra-container penetration loss is generally sufficiently limited to allow for intra-container communication. For the inter-container link, path loss is lowest at $433 \mathrm{MHz}$ in the pathways between container rows, and lowest at $2400 \mathrm{MHz}$ in the small gaps between adjacent containers. Furthermore, path loss models for the extra-container link have been developed for the GSM/UMTS frequencies of 900, 1850, and $2100 \mathrm{MHz}$. Qualitative explanations for the observed frequency- and distance-dependence of path loss have been given. Future work includes testing these assumptions against full-wave simulations of the three container links.

\section{REFERENCES}

[1] D. J. Cichon, and T. Kürner, "Propagation prediction models," in COST 231 Final Report. Brussels, BE: COST Telecom Secretariat, 1999.

[2] S. J. Ambroziak and R. J. Katulski, "On the Usefulness of Selected Radio Waves Propagation Models for Designing Mobile Wireless Systems in Container Terminal Environment," in XXXth URSI General Assembly and Scientific Symposium, Istanbul, TR, Augustus 2011, pp. 1-4.

[3] R. J. Katulski, J. Sadowski, and J. Stefanski, "Propagation Path Loss Modeling in Container Terminal Environment," in 68th IEEE Vehicular Technology Conference (Fall), Calgary, CA, September 2008, pp. 1-4.

[4] C. Xiaohua and X. Hanbin, "Propagation Prediction Model and Performance Analysis of RFID System under Metallic Container Production Circumstance," Microelectronics Journal, vol. 42, pp. 247-252, 2011.

[5] M. Laniel, J.-P. Emond, and A. E. Altunbas, "RFID Behavior Study in Enclosed Marine Container for Real Time Temperature Tracking," Sensing and Instrumentation for Food Quality and Safety, vol. 3, pp. $34-40,2009$.

[6] Y. Jarma, G. Karbaschi, M. Dias de Amorim et al., "Volume-Aware Positioning in the Context of a Marine Port Terminal," Computer Communications, vol. 34, no. 8, pp. 962-972, 2011.

[7] P. S. Hall, Y. Hao, Y. I. Nechayev et al., "Antennas and Propagation for On-Body Communication Systems," IEEE Antennas and Propagation Magazine, vol. 49, no. 3, pp. 41-58, June 2007.

[8] S. Stavrou and S. Saunders, "Factors Influencing Outdoor to Indoor Radio Wave Propagation," in 12th International Conference on Antennas and Propagation, London, UK, March 2003, pp. 581-585. 